\title{
Otto von Helversen, Zoologe aus Passion
}

Hier soll eines Zoologen gedacht werden, der mit ganzer Person und Persönlichkeit Forscher war, der sich auf vielen Gebieten der organismischen und physiologischen Biologie buchstäblich zu Hause fühlte und der es mit Leidenschaft und Hingabe verstand, andere für sein Fach zu begeistern, wenn auch unauffällig, bescheiden und ohne jede Selbstdarstellung. Die Rede ist von Prof. Dr. Otto von Helversen, der am 2. März 2009 tragisch und unerwartet verstarb. Die meisten von uns, vor allem die jüngeren Leser, werden seinen Namen kaum mit arachnologischer Arbeit verbinden, und dennoch hat er wichtige Beiträge geliefert und Anstöße gegeben.

Otto von Helversen, geboren am 9. August 1943 in Sofia/Bulgarien, war schon während seiner Schulzeit begeisterter Zoologe, und auch er gehörte zu den vielen biologischen Forschern, die ihre Karriere mit vogelkundlichen Beobachtungen begannen. In der Umgebung von Wiesbaden, wo er damals wohnte, kartierte er in den Streuobstwiesen Rotkopfwürger, die dort seit Jahrzehnten verschwunden sind. Über die Vögel der Kläranlagen von Schierstein, einem Vorort von Wiesbaden, wo er als Schüler jahrelang beobachtete, veröffentlichte er einen gehaltvollen Beitrag. Und auf seiner ersten Griechenlandreise 1962 - ein Land, dessen Fauna ihn lebenslang fesselte - beobachtete und berichtete er anschließend über das Verhalten von Spornkiebitzen, die erst kurz zuvor in Europa erstmalig als Brutvögel nachgewiesen worden waren. In der Studentenzeit erwachte sein spinnenkundliches Interesse, das er während seines weiteren Lebens beibehielt, wenn es auch nie im Mittelpunkt seiner Forschungen stand. Eine Arbeit über Pseudoskorpione aus dem RheinMain-Gebiet (1966) war Ergebnis der Sammeltätigkeit während des Studiums in Mainz. In einem Aufsatz über die Paarungsstellung bei Webspinnen (1976) zeigte sich sein Interesse, grundlegende $\mathrm{Zu}-$ sammenhänge zu erkunden und zu verstehen. Das galt ebenso für die Homologisierung der Tasthaare bei Pseudoskorpionen, deren Position auf den Pedipalpenscheren wichtige taxonomische Merkmale darstellen. Akribisches Beobachten führte er bis zu mühevoller detektivischer Kleinarbeit. Ihm war aufgefallen, dass Neubeschreibungen von Pseudoskorpionen aus griechischen und jugoslawischen Höh-

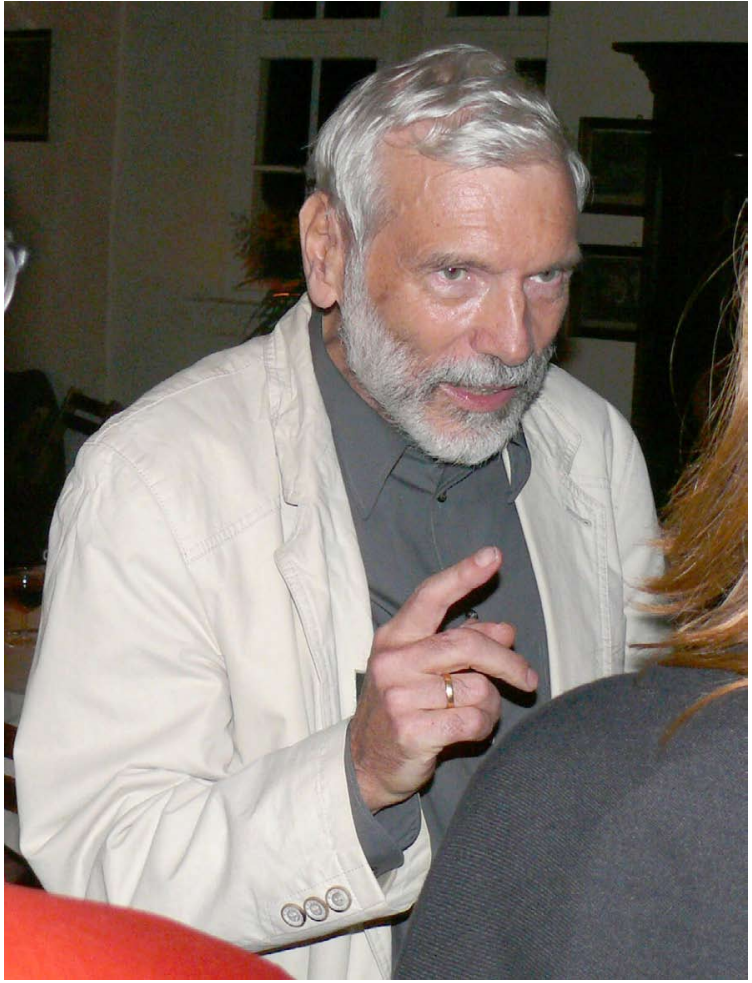

Otto von Helversen bei der Feier zu seinem 65. Geburtstag und zu seiner Emeritierung am 20. September 2008.

Foto: Jan von den Eikel.

len taxonomisch und zoogeografisch nicht „ins Bild“ passten, es schließlich unwahrscheinlich war, dass sie am angegebenen Ort gesammelt worden waren. $\mathrm{Da}$ das gleichzeitig für bestimmte Weberknechte nachweisbar war, ließ sich schließlich der angebliche Sammler des gesamten Materials der unlauteren Berichterstattung überführen, und die „unrichtigen Fundortnennungen" konnten im Einzelnen hiebund stichfest belegt werden (1972). Über Jahrzehnte beschäftigten ihn immer wieder Verhaltensweisen, ob bei Heuschrecken die Physiologie der Lautäußerungen, die Farbwahrnehmung bei Bienen oder der Blütenbesuch bei tropischen Fledermäusen mit allen taxonomischen und physiologischen Komplikationen und Auswirkungen. Ebenso wichtig war ihm die gesamte organismische Zoologie, und er war ein hervorragender Formenkenner heimischer und tropischer Faunen. So faszinierte ihn immer wieder das Paarungsverhalten von Wolfspinnen, in dem er frühzeitig zugleich arterkennende Merkmale wie Fortpflanzungsschranken zwischen 
nahe verwandten Arten ermittelte, die nach morphologischen Merkmalen so gut wie nicht unterscheidbar waren. Seine Schüler dokumentierten die klaren Verhaltensunterschiede der „schwierigen“ mitteleuropäischen Pardosa-Arten, deren Kennung er schon Jahrzehnte zuvor selbst bemerkt und kursorisch untersucht hatte (1990, 2000).

Seinen ersten Studienort Mainz verließ er bald und wechselte nach Tübingen, später nach Freiburg, wo er bei Prof. Bernhard Hassenstein 1970 promoviert wurde und sich 1978 habilitierte. 1979 erhielt er einen Ruf an die Universität Erlangen-Nürnberg, wo er bis zu seiner Emeritierung im Jahre 2008 den Lehrstuhl für Zoologie II leitete.

Seine Möglichkeiten dort nutzte er immer wieder, um engagierte Forscher zusammenzuführen. Im Oktober 1988 lud er zusammen mit Elisabeth Bauchhenß zu einem „Treffen der deutschsprachigen Arachnologen“ ein, auch mit der Absicht, sich wieder mehr, wie er sagte, ,in der Arachnologie bemerkbar zu machen“. Beflügelt durch regen Gedankenaustausch in Erlangen vertieften sich die Kontakte zwischen jungen Spinnenkennern aus Deutschland, Österreich und der Schweiz, und sie begründeten im Herbst 1990 die „Arachnologischen

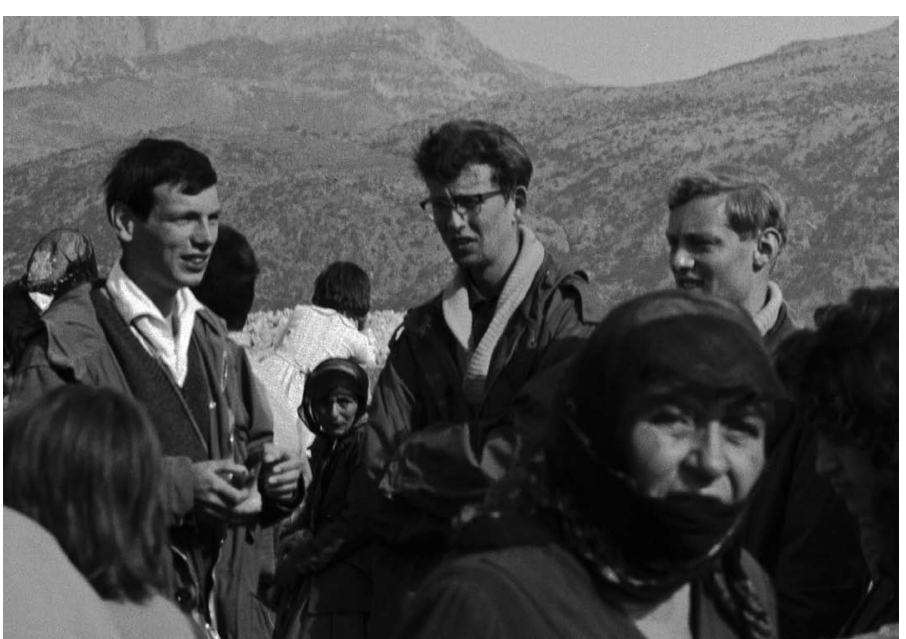

Auf der Reede von Pigadhia, Karpathos, 18. April 1963; von links: Otto von Helversen, Harald Pieper und Ragnar Kinzelbach. - Foto: J. Martens.

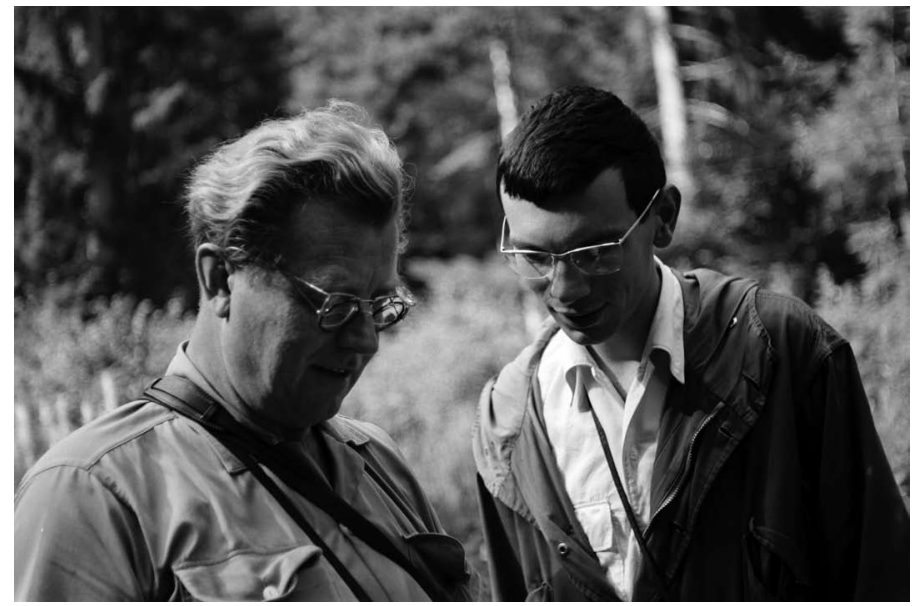

Im Vogelsberg, August 1966. Dr. Vladimír Šilhavý (tschechischer Arzt und Arachnologe; 20. Juli 1913 - 6. Juli 1984) und Otto von Helversen. - Foto: J. Martens.
Mitteilungen“ und die „SARA“ (Süddeutsche Arachnologische Arbeitsgemeinschaft) sowie 1996 die „Arachnologische Gesellschaft“.

An dieser Stelle soll an ein frühes Studienereignis erinnert werden, das für Otto von Helversen und drei seiner damaligen engsten Studienfreunde lebenslange Bestimmung bekam und an dem nachvollzogen werden kann, wie die Laufbahn von Forschern frühzeitig gerichtet und eine Spur gelegt werden kann, die unauslöschliche Auswirkungen hat. Nicht der Einfluss eines akademischen Lehrers ist gemeint, sondern von der befruchtenden und wechselseitigen Begeisterung von Gleichgesinnten soll die Rede sein.
In Ottos erstem Semester fanden sich mit ihm vier gleichgesinnte und auf Taten drängende Mainzer und Giessener Studenten zusammen, ,greenhorns' alle miteinander, die sich für den Plan begeisterten, für Europa neue Tiere zu entdecken. Otto hatte bereits Griechenland-Erfahrung, und so fiel die Wahl schnell auf dieses Land. Die Chance zum Erfolg, so sagten wir uns, läge wohl am Rande Europas am höchsten, so dass wir uns für die ÄgäisInsel Karpathos entschieden - nicht etwa Rhodos. Karpathos gehört geografisch noch zu Europa, Rhodos nicht. Bereits die Anreise war abenteuerlich. Einer der Väter hatte der Gruppe einen alten VW-Bus geschenkt, der nur Vordersitze hatte. Die 
Ladefläche wurde jede Nacht frei geräumt und diente allen Vieren als Nachtquartier. Ein schneller Führerscheinerwerb innerhalb der Gruppe scheiterte - jetzt mussten wir mit nur einem Fahrer starten - Otto! Die Reise begann am 4.3.1963; sie führte über Salzburg, Graz und das damals zwar noch friedliche dafür aber autobahnlose Jugoslawien. Am 9.3. erreichten wir Griechenland und steuerten nach vielen fruchtbaren Einzelexkursionen am 22.3. Athen an. Wo sollte unser wertvolles Auto bleiben? Es mit auf die Insel zu nehmen, war weder bezahlbar noch sinnvoll. Mit leicht beklemmendem Gefühl stellten wir es in einer kleinen Seitenstraße unterhalb des Lykabettos-Hügels mitten in Athen $\mathrm{ab}$... und fanden es nach über drei Wochen unversehrt wieder. Nach eineinhalb Tagen Schiffsfahrt von Piräus aus erreichten wir am 27.3. die Insel Karpathos.

Eine so lange und umsichtig geplante Reise hatten wir versucht, auch fachlich möglichst gut vorzubereiten. Vögel kannten wir alle, Otto war zusammen mit Harald P. schon damals ein ausgewiesener Fledermauskenner, auch die ägäische Herpetofauna war ihnen geläufig. Das genügte uns nicht. Jeder sollte sich zusätzlich um eine Tiergruppe kümmern, eine möglichst umfassende Sammlung anlegen und nach der Rückkehr von der Reise für eine angemessene Bearbeitung sorgen. Das war ganz schön anspruchvoll, aber rückblickend lässt sich sagen, die untereinander zugewiesene Eigenverantwortung wirkte sich überaus segensreich aus. Otto sollte sich (weiterhin) den Pseudoskorpionen widmen, er mit Harald P. zusammen sich um Amphibien und Reptilien kümmern, und dieser allein sich um die Landschnecken. Ragnar K. entschied sich für die Skorpione und wurde darin von allen bestärkt, und Jochen M. fielen die Weberknechte zu. Und überdies - sammeln wollten wir alle Arthropoden, um sie später an Spezialisten zu verteilen. Das brachte uns frühzeitig mit dem SenckenbergMuseum in Kontakt, wo wir bald mit dem Attribut „Karpathos-Clique“ bedacht wurden.

So gerüstet betraten wir die Insel. Zwei von uns mieteten sich im Hafenort Pigadhia ein, die beiden anderen stellten auf den bebuschten Terrassen nicht weit vom Ortsrand ein Zelt auf. Die erste gemeinsame Exkursion am nächsten Morgen durch den regennassen Kiefernwald erbrachte die erste Überraschung und im Grunde bereits die Erfüllung unseres selbst gesteckten Zieles. Frei in der Macchia und im Kiefernwald laufend oder unter Steinen versteckt fanden wir Salamander, farblich unseren Feuersalamandern nicht unähnlich. Wir waren wie erstarrt, denn es war sofort klar - das war der Neunachweis eines Wirbeltieres für Europa. Nie waren auf irgendeiner der ägäischen Inseln Schwanzlurche gefunden worden - und wir entdeckten gleich viele am ersten Tag. Der Widerspruch war für uns schnell geklärt. Alle Herpetologen, die vor uns Karpathos besuchten, Griechen, Österreicher und Italiener, arbeiteten dort im Sommer. Hitze und Trockenheit zu dieser Jahreszeit vermag kein Salamander auszuhalten; sie verkriechen sich in Spalten des Kalkgebirges und halten Sommerruhe. Das war das Glück der Unerfahrenen! Harald P. fand heraus, dass es sich um eine neue Subspezies einer in der SüdwestTürkei verbreiteten Art handelt, die er als $\mathrm{Mer}$ tensiella luschani helverseni Pieper, 1963 beschrieb. Genetische Studien ergaben inzwischen, dass die Population von Karpathos eine eigene Art darstellt und sogar eine neu aufgestellte Gattung vertritt, Lyciasalamandra Veith \& Steinfartz, 2004. Wir durchstreiften die Insel reichlich drei Wochen lang, bestiegen das Schiff am 18.4. und erreichten am 26.4. wohlbehalten Mainz.

Alle Teilnehmer dieser Reise stellten immer wieder fest, dass dieses selbst organisierte und umsichtig geplante Unternehmen für jeden Einzelnen ein wichtiges Erlebnis war, das einen guten Teil der späteren wissenschaftlichen Laufbahn entscheidend prägte. Es darf dabei nicht verkannt werden, dass Otto von Helversen ganz maßgeblich an der gedanklichen Strukturierung dieser Reise Anteil hatte - und die alleinige Fahrleistung erbringen musste. Alle vier blieben über die Jahrzehnte miteinander in Kontakt, und das Forschen in fremden Ländern war uns frühzeitig vorgezeichnet. Vor allem Ottos ökologische Arbeiten an Fledermäusen führten ihn regelmäßig nach Mexico, Kuba und Costa Rica. Als eine erneute Reise nach Costa Rica in diesem Frühjahr anstand, setzte ein ärztlicher Kunstfehler allen weiteren Plänen ein Ende.

Wir verneigen uns vor dem Freund und einer großen Forscherpersönlichkeit.

Jochen Martens, Harald Pieper \& Ragnar Kinzelbach 


\section{Arachnologische Veröffentlichungen von Otto von Helversen}

HeLversen O. v. (1965): Scientific expedition to the Salvage Islands. July 1963. VI. Einige Pseudoskorpione von den Ilhas Selvagens. - Boletim do Museu Municipal do Funchal 19: 95-103

Helversen O. v. (1966): Pseudoskorpione aus dem Rhein-Main-Gebiet. - Senckenbergiana biologica 47: $131-150$

Helversen O. v. (1966): Über die Homologie der Tasthaare bei Pseudoskorpionen (Arach.). - Senckenbergiana biologica 47: 185-195

HeLVERSEN O. v. (1968): Troglochthonius doratodactylus n. sp., ein troglobionter Chthoniide (Arachnida; Pseudoscorpiones: Chthoniidae). - Senckenbergiana biologica 49: 59-65

Helversen O.v. (1969): Roncus (Parablotbrus) peramae n. sp., ein troglobionter Neobisiide aus einer griechischen Tropfsteinhöhle (Arachnida; Pseudoscorpiones: Neobisiidae). - Senckenbergiana biologica 50: $225-233$

Helversen O. v. \& K.H. HARMS (1969): Zur Spinnenfauna Deutschlands, VII. Für Deutschland neue Wolfspinnen der Gattungen Pirata und Pardosa (Arachnida: Araneae: Lycosidae). - Senckenbergiana biologica 50: 367-373

Helversen O. v. \& J. MARTEns (1971): Pseudoskorpione und Weberknechte. In: SAUER K.F.J. \& M. SCHNETTER (Hrsg.): Die Wutach. Naturkundliche Monographie einer Flußlandschaft. Badischer Landesverein für Naturkunde und Naturschutz, Freiburg im Breisgau. - Die Natur- und Landschaftsschutzgebiete Baden-Württembergs 6: 377-385

Helversen O. v. \& J. MARTENS (1972): Unrichtige Fundort-Angaben in der Arachniden-Sammlung Roewer. Senckenbergiana biologica 53: 109-123

Helversen O. v. (1974): Die Wolfspinnen (Lycosidae) im Schutzgebiet „Taubergießen“. In: Landesstelle für Naturschutz und Landschaftspflege Baden-Württemberg (Hrsg.): Das Taubergießen. Eine Rheinauenlandschaft. - Die Natur- und Landschaftsschutzgebiete Baden-Württembergs 7: 547-549

HELVERSEN O. v. (1976): Gedanken zur Evolution der Paarungsstellung bei den Spinnen (Arachnida: Araneae). - Entomologica Germanica 3: 13-28

Gack C. \& O. v. HeLversen (1976): Zum Verhalten einer gynandromorphen Wolfspinne (Arachnida, Araneae, Lycosidae). - Entomologica Germanica 3: 109-118
BARTHEL J. \& O. v. HELVERSEN (1990): Pardosa wagleri (Hahn 1822) and Pardosa saturatior Simon 1937, a pair of sibling species (Araneae, Lycosidae). - Bulletin de la Société européenne d'Arachnologie, hors série 1: $17-23$

CORDES D. \& O. v. HELVERSEN (1990): Indications for the existence of Alopecosa barbipes (Sundevall 1832) as a sibling species' to Alopecosa accentuata (Latreille 1817) - results of morphological, ethological and biogeographical studies. - Bulletin de la Société européenne d'Arachnologie, hors série 1: 70-74

Töpfer-Hofmann G. \& O. v. Helversen (1990): Four species of the Pardosa lugubris-group in Central Europe (Araneae: Lycosidae) - A preliminary report. - Bulletin de la Société européenne d'Arachnologie, hors série 1: 349-352

BauchHenss E., C. GaCK, K.H. Harms, O. v. HeLVERSEN, A. KOBEL-LAMPARSKI \& J. WUNDERLICH (1993): „Workshop zur Taxonomie mitteleuropäischer Spinnen“ in Erlangen (8.-10.01.1993): Taxonomie und Faunistik der kleinen Euophrys-Arten und der Gattungen Neaetha und Pellenes (Salticidae). - Arachnologische Mitteilungen 5: 52-53

TÖPFER-HOFMANN G., D. CORDES \&O.v. HELVERSEN (2000): Cryptic species and behavioural isolation in the Pardosa lugubris group (Araneae, Lycosidae), with description of two new species. - Bulletin of the British arachnological Society 11: 257-274

\section{Von Otto von Helversen neu beschriebene Spinnentier-Taxa}

\section{Pseudoscorpiones}

Diplotemnus pieperi v. Helversen, 1965 (Pitão Grande, Ilhas Selvagens, Portugal)

Garypus saxicola salvagensis v. Helversen, 1965 (Ilheu de Fora, Ilhas Selvagens, Portugal)

Troglochthonius doratodactylus v. Helversen, 1968 (Sardinien, Italien; genauer Fundort unbekannt)

Roncus peramae v. Helversen, 1969 (Perama bei Joannina, Epirus, Griechenland)

\section{Araneae}

Pardosa pertinax v. Helversen, 2000 (Vernon Gebirge, Griechenland) 